\title{
Implementing New Advanced Learning Scenarios Through GRID Technologies
}

\author{
Matteo Gaeta ${ }^{*} \ddagger$, Pierluigi Ritrovato ${ }^{*} \ddagger$, Saverio Salerno ${ }^{\dagger *}$ \\ ritrovato@crmpa.unisa.it; gaeta@crmpa.unisa.it; salerno@unisa.it \\ *Consorzio CRMPA - Centro di Ricerca in Matematica Pura ed Applicata \\ ${ }^{\dagger}$ Dipartimento di Ingegneria dell'Informazione e Matematica Applicata - Università di Salerno \\ ${ }^{\ddagger}$ Centre of Excellence "Methods and Tools for Learning and Knowledge"- Università di Salerno \\ Via Ponte don Melillo - 84084 Fisciano - ITALY
}

Keywords: analysing the economic, technological and pedagogical challenges underpinning the use of GRID technologies for e-Learning

\section{POSITION PAPER}

In this paper we present our vision concerning the use of GRID technologies for the implementation of the next generation of learning solutions and in particular for supporting distance learning. We believe that the new requirements that new Learning environment should satisfy could transform these solutions into killing applications for GRID technologies.

Our ideas move essentially from two main thoughtfulness: from one side there are the lacks characterising the existing software platforms for supporting traditional and distance learning processes and, from the other side there is the continuous achievement of GRID technologies as a solution for truly implementation of e-science infrastructure.

The main drawbacks of current distance learning solutions are twofold: they only support a part of the whole learning process (the simple one), and how ICT is used.

Concerning the former issue, many of the market-available e-learning solutions are focused on supporting only a specific aspect of the learning process notably the content delivery (content centred approach). Nowadays is emerging a strongly need for supporting other fundamental aspects of the learning process such as initial skill assessment, learner progress metering through definition of specific learning goals, formal representation of the knowledge to be transferred to the learner (metadata standards, ontology, taxonomy, etc), automatic discovering and self-adaptation to individual learning styles, continuous assessment, etc.

This lack is mainly due to the fact of considering the didactical communication as the information transfer from the addresser (teacher) to an addressee (student), where the propaedeutic (preparatory) mean is a written text.

This has a direct impact on the latter issues. In fact, most used ICT technologies in learning are the more traditional one such as video (TV programmes, videoconferencing sessions, web casting events) and in some cases traditional CBT enriched with multimedia contents.

It is clear to everybody that technology is not a "panacea" for all the educational ills, but today's technologies are essential tools of the teaching trade and are essential for the achievement of a breakthrough in this field. As showed by several EU and US studies the new research paths are moving towards supporting the whole learning process for individuals and organisations facilitating 
the representation, acquisition and sharing of knowledge and skills in any place, at any time and any pace.

This implies the need of new advanced solution allowing the implementation of user centred collaborative environment for ubiquitous learning (CSCL - Computer Supported Collaborative Learning) through a fully exploitation of emerging networking facilities (e.g. broadband and wireless) simplifying the access to advanced software solutions (simulation environments, 3D visualisation systems, digital libraries, etc.).

In this frame, the term "ubiquitous" is not strictly referred to geographic location but it means the ability to support multiple learning contexts and automatically adapt to them.

This more wide vision of ubiquitous learning means that these new environments should support, at the same time, new emerging pedagogical approaches such the one based on inductive-simulative and the experience models facilitating the construction of personalized learning paths.

Although Internet is perhaps the most transformative technology in history, reshaping business, media, entertainment, and society in an astonishing way, it is just now being tapped to transform education. Nowadays, Standard Internet technologies, due to their data oriented nature, have well supported the implementation and exploitation of traditional content based on learning solutions. A lot of efforts are currently undergoing for defining standards for learning content representation, and their interoperability.

Now, as for the learning solutions a shift is needed for Internet technologies.

GRID technologies are rising as the next generation of Internet. These technologies promise to do what the internet has done with data for the applications. GRID technologies define a new powerful computing paradigm by analogy to the electric Power Grid. In this vision, a customer of the GRID will be able to use his or her private work place (Workstation, PC, UMTS phone....) to invoke any application from a remote system, use the system best suited for executing that particular application, access data securely and consistently from remote sites, exploit multiple systems to complete complex tasks in an economical manner, or use multiple systems to solve large problems that exceed the capacity of a single one.

Another interesting aspect of GRID technologies is their support for resource sharing and problem solving in dynamic, multi-institutional virtual organizations. In this vision, the sharing doesn't mean simply exchange of data or files but rather a concrete access to resources (e.g. computers, software, data, etc.). This "sharing capability" imposes the definition and implementation of welldefined resource management policies to specify what is accessible, from whom and under which conditions. A set of individuals and/or institutions defined by such sharing rules form what is called a Virtual Organization (VO).

This sharing capability and support for VO implementation has been one of the main key success factors of GRID as enabling technology for e-science infrastructure.

This kind of VO implemented using GRID technologies seems to provide the right answers to the needs rising from the emerging ubiquitous learning solutions.

Actually, the Grid could be used as a technology glue (seamless network combining wireless and broadband network, advanced software solutions for personalised mobile learning), providing the user with a uniform way to access these resources by means of several kinds of devices.

These technologies will provide, in a natural way, a support for both distance and traditional learning activities facilitating the experimentation and evaluation of new emerging didactical models based on the learning by doing approach. 
In order to support these new scenarios it is important that GRID move from a niche technology to a commodity one. Fortunately, it seems that the new research directions for the development of GRID technologies are moving towards that way. In deed, in the framework of the Global Grid Forum (the official organism for managing GRID standards) many efforts have been devoting to the definition of the OGSA (Open Grid Service Architecture) that essentially defines a common extensible framework for Grid services specification. The Grid Services will be accessible through web service standard mechanism.

This standardized approach allows, from a one hand, to create new GRID services need for the learning domain (GRID domain specific) and, on the other, to integrate existing solutions making them accessible through the GRID, facilitating the interoperability from the existing learning solutions that is actually a real issue in this field.

Although the GRID technologies and their development seem to go towards the right direction for the implementation of new and advanced learning solutions, as for all new ideas plenty of work is still required.

In particular, this effort should be oriented to:

- the definition of methodologies and techniques for making grid-aware existing applications (in order to preserve existing investments)

- the definition of learning GRID services

- solutions usability and their further integration with the new ambient intelligent user interface. 